\title{
The management of Bochdalek congenital diaphragmatic hernia: a single center experience and review of the literature
}

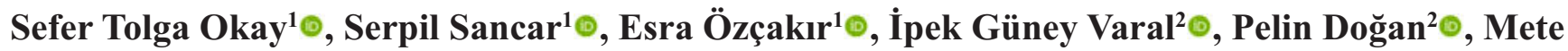 \\ Kaya $^{1} \odot$
}

${ }^{1}$ Department of Pediatric Surgery, University of Health Sciences, Bursa Yüksek Ihtisas Training and Research Hospital, Bursa, Turkey ${ }^{2}$ Department of Neonatalogy, University of Health Sciences, Bursa Yüksek Ihtisas Training and Research Hospital, Bursa, Turkey

\begin{abstract}
Objectives: Congenital diaphragmatic hernia $(\mathrm{CDH})$ is a rare congenital anomaly of the diaphragm with pulmonary hypoplasia and persistent pulmonary hypertension and has serious consequences. Despite recent diagnosis and treatment strategies, $\mathrm{CDH}$ is still a challenging condition. We aim to present our clinical experiences of $\mathrm{CDH}$, and review of the literature.

Methods: Data of CDH patients who were operated in our clinic between January 2010 and September 2018 were obtained from the patient's chart. The clinical course and results of the patients with Bochdalek type CDH were reviewed.

Results: We performed diaphragmatic closure in 16 patients with Bochdalek CDH during study period. Fourteen $(82 \%)$ cases were diagnosed antenatally. In $5(29 \%)$ patients, preoperative pulmonary hypertension developed and nitric oxide was administered. Twelve of the sixteen patients $(75 \%)$ underwent primary repair and 4 of them underwent prosthetic patch. Three patients were repaired thoracoscopically. Five (31\%) patients died due to severe persistent pulmonary hypertension on postoperative period. There was no recurrence in our patients who were followed-up for a mean of 27 months.

Conclusion: In the management of Bochdalek $\mathrm{CDH}$, the clinical success has been increasing in parallel with the important developments with the application of new treatment modalities in the neonatal intensive care units and new surgical techniques in recent years.
\end{abstract}

Keywords: Bochdalek hernia, congenital diaphragmatic hernia, newborn, pulmonary hypertension

Copt ongenital diaphragmatic hernias $(\mathrm{CDH})$ occur in approximately 2500-3000 births and they are congenital anomalies. Diaphragm begins to develop at the fourth weeks of the pregnancy, and completes the formation in about 20 weeks. It has been found that some genetic and environmental factors play a role in the pathogenesis, but whose exact pathogenesis is still not understood well. Anatomically, $\mathrm{CDH}$ can be clas- sified as posterolateral (Bochdalek, 70-75\%), anterior (Morgagni, 23-28\%) or central (2-7\%) CDH caused by defects. Bochdalek hernias are the most common type of congenital diaphragmatic hernia resulting from a failure of pleuroperitoneal membrane closure in utero, characterized by the protrusion of intra-abdominal contents into the thoracic cavity through the posterolateral diaphragmatic defect. In $85 \%$ of the cases, 
Bochdalek $\mathrm{CDH}$ is seen on the left side, in 13\% right and in 2\% bilaterally [1-3].

In Bochdalek $\mathrm{CDH}$ patients, pulmonary hypoplasia and pulmonary hypertension $(\mathrm{PH})$ are the major determinants of survival in the neonatal period and predictors of long-term morbidity. Because of pulmonary hypoplasia and $\mathrm{PH}$ which are the results of preventing the development of terminal bronchioles, alveoli and pulmonary vessels, critical respiratory insufficiency arises immediately after birth. Although many pharmacological treatments and also many current applications such as "gentle ventilation", high frequency oscillatory ventilation (HFOV) and extracorporeal membrane oxygenation (ECMO) are now used, the mortality and morbidity rates are still high in Bochdalek CDH patients. Additionally, there are still ongoing discussions on some aspects of this disorder such as the best method of surgical repair, the choice of patch to be used in place of lacking diaphragm and optimal time of surgery $[2,4]$.

In this study, we aimed to retrospectively review the patients who underwent surgery for Bochdalek $\mathrm{CDH}$, and to present our approaches in the light of the literature.

\section{METHODS}

Between January 2010 and September 2018, data of newborns who were operated in our clinic because of Bochdalek $\mathrm{CDH}$ have been reviewed retrospectively. The cases have been evaluated from the antenatal findings, gestational ages, birth weights, ventilator requirements and parameters, preoperative data, additional medical treatments, operation times, operative pattern, patch requirement, duration of the mechanical ventilation, complications, additional surgical procedures and mortality.

In our hospital, neonatal intensive care unit (NICU) is managed by neonatologists. Pre- and postoperative care of the patients with Bochdalek $\mathrm{CDH}$ are implemented in this unit. Although the patients with Bochdalek $\mathrm{CDH}$ are managed by different neonatologists, standard approaches are carried out. The management of Bochdalek CDH patients in our institution is briefly: postnatally, after initial resuscitation with avoidance of the bag mask ventilation and subsequent barotrauma, a nasogastric tube is placed, a suitable vascular access is inserted, and the case with Bochdalek CDH is transferred to the NICU. The patient is monitored for pre-/ and postductal $\mathrm{O}_{2}$ saturation. Arterial blood gas and initial $\mathrm{CBC}$, basic metabolic parameters, and coagulation profile is measured. A chest X-ray and abdominal Xray (Fig. 1), ultrasonography (US), and echocardiography to investigate accompanying organ anomalies, and to assess the degree of pulmonary hypertension, ductal patency and ventricular function are studied routinely. Intravenous fluid, electrolyte and nutrition are administered. Analgesics and sedatives are also administered to facilitate optimal ventilation especially in neonates with severe pulmonary hypertension. Synchronized conventional ventilation (SIMV) with tidal volume monitoring is chosen to allow permissive hypercapnia as initial ventilatory strategy. If needed, high frequency oscillatory ventilation (HFOV) which is a lung protective strategy is used to reduce ventilator induced lung injury. If $\mathrm{PH}$ persists, pulmonary vasodilator therapy is started, with

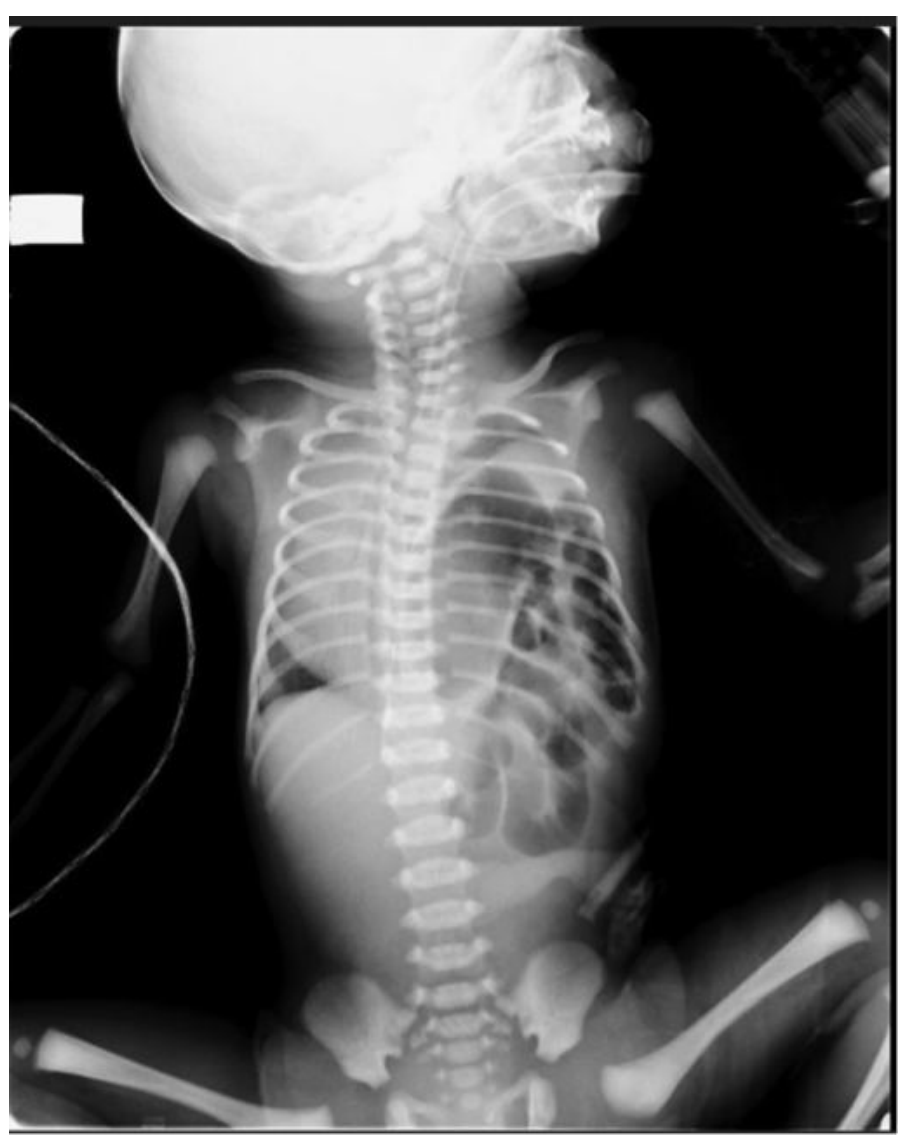

Fig. 1. The chest X-Ray showing the large diaphragmatic hernia. 


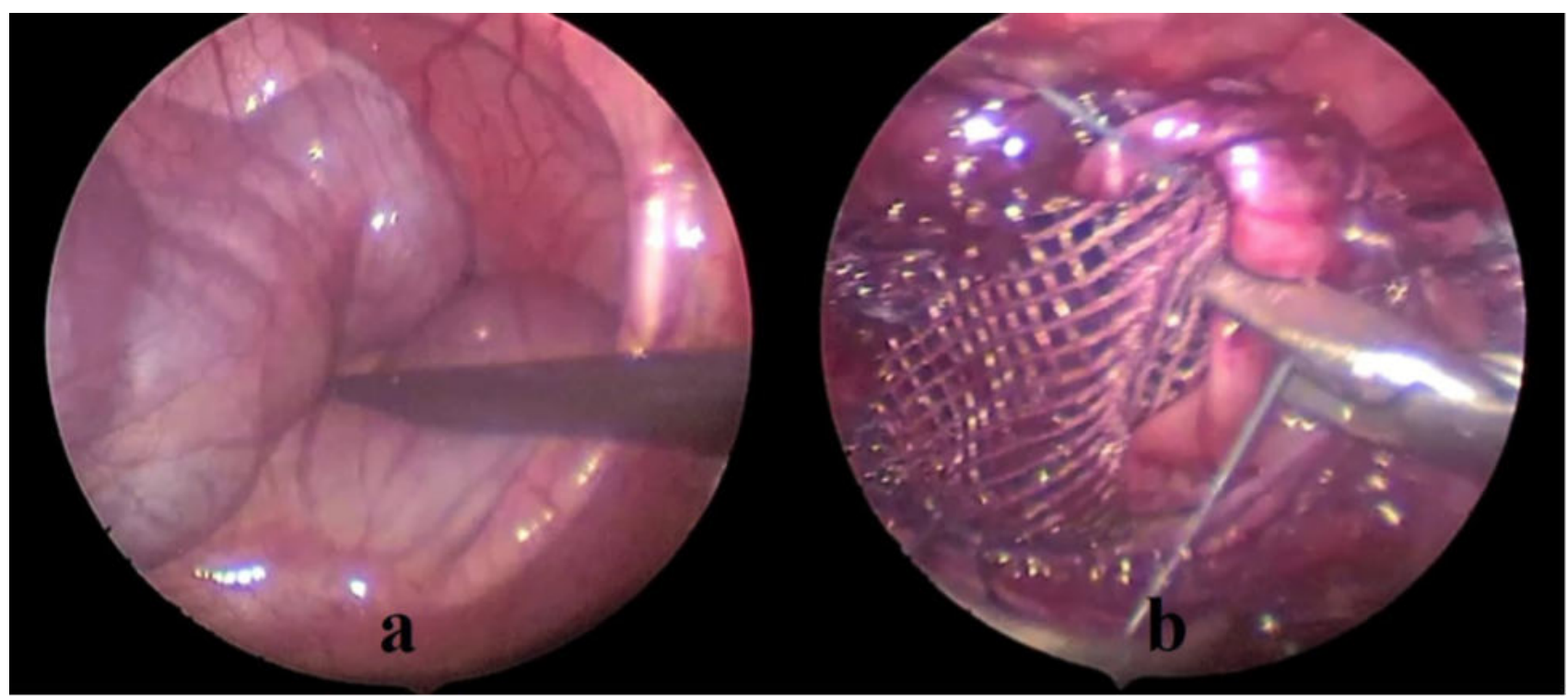

Fig. 2. Intraoperative photos showing the diaphragmatic defect which is too large to close with thoracoscopic primary repair (a), and using prosthetic patch to repair diaphragmatic hernia in same patient (b).

inhaled NO as the first choice and/or Sildenafil a phosphodiesterae-5 inhibitor. ECMO in the treatment of neonates with Bochdalek $\mathrm{CDH}$ is not currently used in our institution.

For surgical repair, laparotomy was mostly performed with the transverse incision of the classical left upper quadrant. After gently pulling the organs into the abdomen, the defect margins were primary closed with U-shaped suturing with nonabsorbable sutures. If the diaphragmatic edges were not developed, the defect was closed by using either of the muscle flaps prepared from the near structures or synthetic patch in very large defects. Finally, the organs were checked for other anomalies and the intestinal malrotation before the abdomen was closed. If the abdominal cavity is not sufficient, only skin closure can be done, but it was not needed in our cases.

Table 1. Antenatal and postnatal clinical data of patients with CDH

\begin{tabular}{|c|c|}
\hline Gender (F/M) & $10 / 7$ \\
\hline Antenatal diagnosis $(\%)$ & $14(82 \%)$ \\
\hline $\begin{array}{l}\text { Mean gestational age at } \\
\text { diagnosis }\end{array}$ & 20 weeks (16-32 weeks) \\
\hline Mean gestational age at birth & 35 weeks (30-41 weeks) \\
\hline Mean birth weight & 2810 gr (1100-4400 gr) \\
\hline
\end{tabular}

In the thoracoscopic repairing, the optic port under the scapula and two work ports from the laterals of this point were placed, herniated organs pushed to the abdomen and the defect was closed with either primary U-shaped sutures or synthetic mesh (Fig. 2).

\section{RESULTS}

During the 8-year study period, data of 17 patients were found and restorative surgeries were performed in 16 patients for Bochdalek CDH. The antenatal and postnatal data of the cases are presented in Table 1. Fourteen out of 17 cases (82\%) were diagnosed antenatally, and the mean gestational age for diagnosis was 20 weeks (min-max: 16- 32). In one case, the right Bochdalek CDH was detected (6\%), and this case was being followed conservatively since the liver prevented intestinal herniation. Due to the development of $\mathrm{PH}$, NO was initiated in 5 patients (29\%) preoperatively. A patient with very low birth weight and bilateral severe pulmonary hypoplasia died before surgery. Patients were taken to the operation approximately in postpartum 30th hours. The records of the operations and postnatal follow-up are summarized in Table 2. Primary repair was made with laparotomy in 12 patients. Also, in one infant, primary repair was made by forming flaps from intercostal and 
latissimusdorsi muscle flaps. Synthetic patch was required in four patients. Mostly, propylene mesh was used as the patch. Besides propylene mesh, polytetrafluoroethylene (PTFE), polyglactin and propylene mixture meshs were also used as the patch. Three cases were approached with thoracoscopic method, primary closure of the defect was achieved in two patients, and propylene mesh was implanted in one. The mean duration of the mechanical ventilation was 5.5 days. Because of persistent $\mathrm{PH}$, NO was continued or started in 7 patients postoperatively.

Table 2. Surgical and postoperative follow-up symptoms of patients with CDH

The timing of surgery after $30^{\text {th }}$ hour $\left(24^{\text {th }}-52^{\text {nd }}\right.$ hour $)$
birth

Operation

16

\begin{tabular}{|c|c|}
\hline \multicolumn{2}{|l|}{ Laparotomy } \\
\hline Primary & 10 \\
\hline Prosthetic & 3 \\
\hline \multicolumn{2}{|l|}{ Thoracoscopic } \\
\hline Primary & 2 \\
\hline Prosthetic & 1 \\
\hline
\end{tabular}

\section{Hernia content}

\section{Liver}

Spleen

Stomach

Small bowel

Colon

Additional intraabdominal anomaly

Mean duration on ventilator support

Long term morbidity

$\operatorname{GER}(\mathrm{n}=2)$

Pectus excavatum $(n=3)$

Additional surgery

Fundoplication $(\mathrm{n}=2)$

Mortality

$31 \%$

$\mathrm{CDH}=$ Congenital diaphragmatic hernia, GER = gastroesophageal reflux

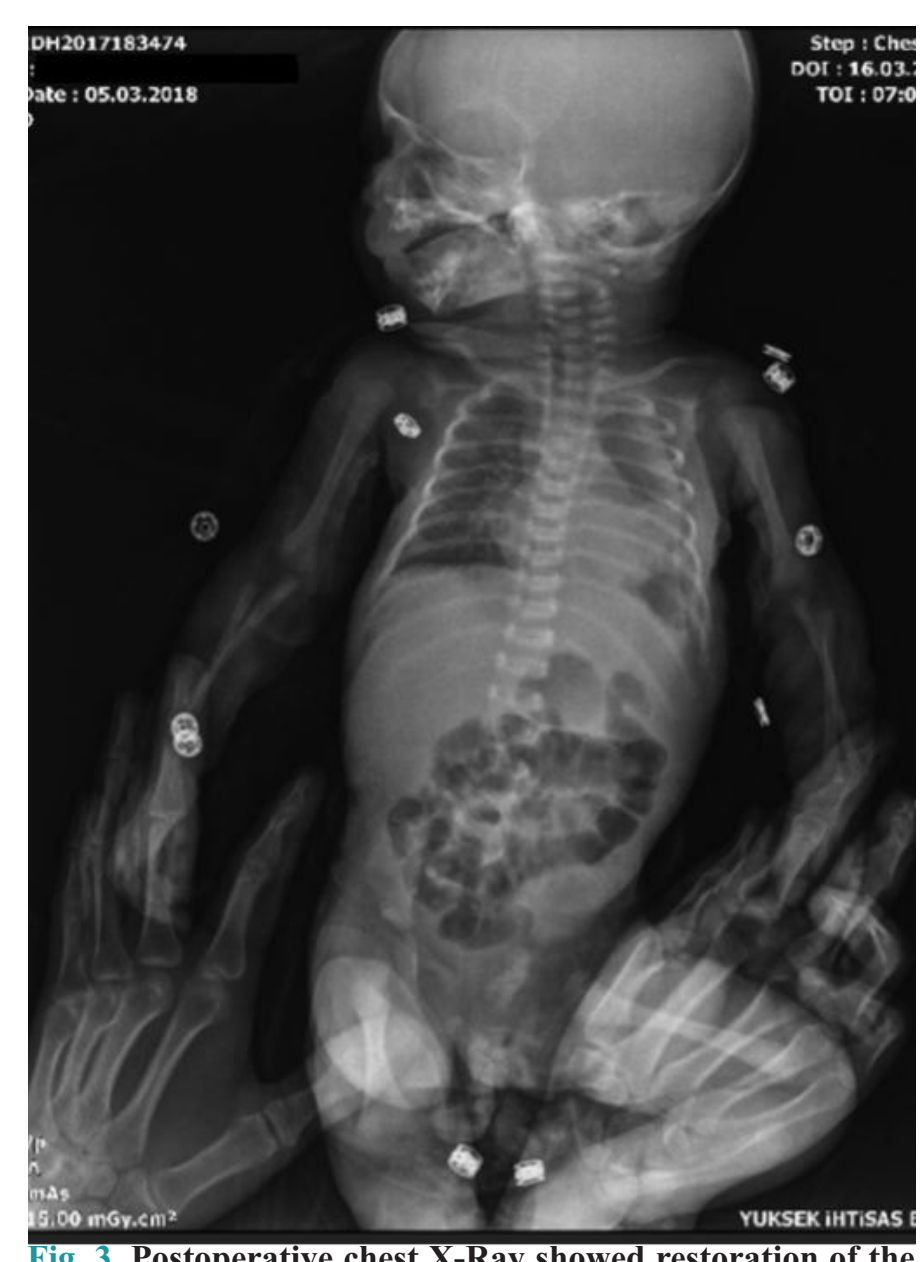

Fig. 3. Postoperative chest X-Ray showed restoration of the normal contour of the left hemidiaphragm in the patient who underwent thoracoscopic repair.

\section{Sildenafil was initiated in five patients.}

Five patients died due to postnatal early period persistent $\mathrm{PH}$ and respiratory insufficiency (31\%). Mean follow-up period was 27 months (1-84 months). Hernia recurrence was not seen in any patient (Fig. 3). However, later on, two patients were re-operated with the cause of severe gastroesophageal reflux (GER).

\section{DISCUSSION}

In recent years, despite the important developments in surgery and anesthesia of newborn, the management of Bochdalek CDH patients is still a major problem. There are significant differences between clinics in the use of antenatal steroids, ventilation modes, methods used in $\mathrm{PH}$ treatment, the usage of ECMO, and timing of surgical repair [4]. 
These are the important issues striving to be standardized. The findings detected on the antenatal US and fetal magnetic resonance imaging (MRI) has begun to be used as prognostic criteria in Bochdalek $\mathrm{CDH}$. It has been suggested that a prediction about prognosis can be made according to observed/expected lung-to-head ratio, total lung volume, liver herniation grade, and appearance of the stomach over four chambers of the heart in US and fetal MRI. The reason for the use of lung-head ratio is an indirect evaluation of the contralateral lung volume and therefore the possibility of pulmonary hypoplasia. The degree of volumetric intrathoracic liver herniation using fetal MRI indicates that the liver is better on lower position at predicting postnatal survival than the above [5]. In our series, Bochdalek CDH was detected in the antenatal US follow-up of 14 patients. However fetal MRI was not performed in our patients. It is obvious that there is a need to establish the standards in the antenatal screening of Bochdalek $\mathrm{CDH}$.

Many studies have been also done on the prediction of the survival rates of newborns with Bochdalek CDH postnatally. Prognosis is determined by defect size, patch requirement, pulmonary hypoplasia, pulmonary vascular structure and $\mathrm{PH}$ grade. Recently, a model has been tried to be used to estimate the probability of survival based on various parameters such as low birth weight, low Apgar score, severe $\mathrm{PH}$, major cardiac anomaly and chromosome anomalies [6]. This model seems that it could be implemented with data that could easily be found in the clinical setting. We did not have sufficient data on the postnatal and preoperative pulmonary morphology in our series.

In order to reduce pulmonary vascular resistance and right-to-left shunt preoperatively, alkalosis $(\mathrm{pH}>$ 7.55; PCO2 $<20 \mathrm{mmHg}$ ), which was attempted to be induced by chemical and ventilator settings, led to barotrauma and later neurological sequel. Thereupon, "permissive hypercapnia" is defined. Thus, it has been proposed that HFOV, which is the most appropriate ventilator mode for optimal ventilation, should be administered initially, as it is more appropriate than conventional mechanic ventilation strategies [7]. The most frequently used ventilator mode was SIMV as traditional mechanical ventilation mode in our patients on preoperative period. HFOV administration was initiated in the patients in the early period if required.
$\mathrm{PH}$ is a pathological condition of the pulmonary vascular structure and results in pathophysiological pulmonary circulation. $\mathrm{PH}$ generally affects oxygenation, ventilation, and/or cardiac function. In patients with Bochdalek $\mathrm{CDH}, \mathrm{PH}$ is caused by vascular thickening of the medial and adventitial layers of pulmonary vascular structures, a hypoplastic vascular bed with diminishing branching, and pulmonary artery/arteriole which have the inappropriate response to physiological and pharmacological signals. As a result of the external compression of the herniated organs into the lung, despite the high oxygen supply, the $\mathrm{O}_{2}$ saturation cannot be increased and carbon dioxide retention occurs [8]. On the other hand, it was shown that mechanisms such as retinoic acid pathway, NO pathway, endothelin pathway, and vascular endothelial growth factor contributes to the formation and/or progression of the $\mathrm{PH}$ in the patients with Bochdalek $\mathrm{CDH}$. It has been shown that nitrophen, a retinal dehydrogenase inhibitor, forms Bochdalek $\mathrm{CDH}$ experimentally. It was also seen that Bochdalek $\mathrm{CDH}$ risk increased in infants of mothers who have received less than $800 \mathrm{mg}$ of vitamin A during pregnancy [9]. NO pathway plays a role in angiogenesis, lung development and vasorelaxation in the human fetal lung. NO is spread to smooth muscle cells from endothelia and decrease the cytosolic calcium depending on c-GMP, and induces pulmonary vasodilation. In fact, endothelial NO synthase, an enzyme responsible for the expression of NO in the vascular endothelium, both increased and decreased in animal and human Bochdalek CDH studies. For this reason, despite the responsibility for $\mathrm{PH}$ in patients with Bochdalek $\mathrm{CDH}$ is still not understood completely, but NO continues to be used in $\mathrm{PH}$ management. In the etiology of $\mathrm{PH}$, besides endothelin-1, which is highly detected in Bochdalek $\mathrm{CDH}$ patients, the disorder of balance ETA (vasoconstriction) and ETB (vasodilatation) which primary receptors of endothelin-1, have been responsible for increased pulmonary vascular pressure and exacerbation [10]. Lastly, vascular endothelial growth factor has been experimentally found at different levels in nitrophen-induced Bochdalek $\mathrm{CDH}$. It has been found to stimulate pulmonary vasculogenesis/angiogenesis which is critical in embryological pulmonary development and growth. 
On the other hand, human postmortem studies have shown that this factor has been found to be increased, and it has been estimated that it is probably due to a response to the stimulation of angiogenesis in the hypoplastic vascular structure [8,11]. In the examination of our patients who were included in our study, the intake of vitamin A was not given details from history. However, we know that pregnant women followed by obstetricians routinely receive multivitamin pills. Although studies on endothelin and vascular growth factor levels were not measured, preoperative and postoperative NO treatments were applied to three patients. ECMO, which is a subject in itself in $\mathrm{PH}$ management, is seen as the last step treatment of $\mathrm{PH}$, and is an invasive approach. Additionally, besides NO inhalation, many drugs such as sildenafil, milrinone, prostanoids, prostoglandin E1, bosentan and $\mathrm{MgSO}_{4}$, are applied in $\mathrm{PH}$ management. Sildenafil use is recommended to be stored in refractive $\mathrm{PH}$ cases. And, it is claimed that it connects with the better results. On the other hand, in 2015, updated CDH EURO Consortium in order to provide postnatal treatment is standardized in European countries has been proposed in consensus that, however, if there is no or an insufficient response to inhaler NO, intravenous prostacyclin, intravenous sildenafil or medication involving the endothelin pathway should be considered [12]. Sildenafil was begun to use in five patients in our series.

There is no consensus on the timing of surgery for the patients with Bochdalek CDH. Some centers promote early surgery (first 24 hours). Over the time, this approach has largely changed from early surgical intervention to management and control of pulmonary hypertension before surgical repair [1]. The European working group suggests that surgical repair must be performed after physiologic respiratory and cardiac stabilization [12]. We also operated our cases at the postnatal $30^{\text {th }}$ hour.

Standard open surgery in Bochdalek $\mathrm{CDH}$ patients is performed using a subcostal incision; the diaphragm is repaired by using with interrupted non-absorbable sutures after returning the herniated organs to the abdomen from thoracic cavity. In the majority of cases (60-70\%), primary closure of the diaphragm can be achieved. Although it is known to be unsuccessful in half, in cases where the diaphragm never develops or the defect is too large to close the defect, muscle flap techniques, a synthetic or biosynthetic prosthetic patch may be required [13]. The muscle flaps have the advantage of repairing the diaphragm without tension, but are often impractical for primary repair. Abdominal compartment syndrome is rare after Bochdalek CDH repair. But, if there is not enough abdominal cavity, the closin of the abdominal fascia can be delayed [14]. After Silen and his colleagues performed the first thoracoscopic approach in 1995, minimally invasive surgery (MIS) arose as a safe and feasible alternative to open surgery [15]. The benefits of MIS include reduced postoperative pain and a good cosmetic appearance. Beside these benefits, MIS provides reduction of organs within a wide field of view through thoracic cavity. The disadvantages of MIS are limitation of diagnosing and treatment of other intraabdominal pathologies such as malrotation, narrow space for diaphragm repair, and physiological sensitivity of the newborn [13]. In our series, the diaphragmatic defects were closed with primary sutures in 12 patients (75\%), and two of whom were thoracoscopic. The intercostal and latissimusdorsi muscle flaps were used to close the defect in one. In four cases, prosthetic material was needed to close the large diaphragmatic defect, and the most common propylene mesh patch was used in these patients. The patch was placed thoracoscopically on one of these patients. The malrotation anomaly was detected and Ladd Procedure performed in two patients who operated by laparotomy. The abdominal compartment was not seen in any patient. None of the patients developed recurrence.

Mortality rates for Bochdalek $\mathrm{CDH}$ patients vary in the literature. While survival rates were $90 \%$ in single-center studies, the general mortality rate in multicenter studies ranged from 15 to $70 \%[1,2,5,6]$. This difference makes it difficult to accurately assess the effect of recent treatment progress on survival outcomes. In our series, the overall survival rate is $69 \%$. This shows that it is compatible with the literature. The success of surgical treatment may be related to patient density. In the hospital complex which includes our center, there are big birth clinic and the most equipped newborn intensive care unit of the region. These provide that the patients are gathered at our center.

Surviving Bochdalek $\mathrm{CDH}$ patients in the long term expose to the risk of many diseases. These 
chronic diseases include chronic pulmonary diseases such as restrictive pulmonary defects, reactive airway; gastrointestinal morbidity such as GER (45-90\%); musculoskeletal abnormalities such as chest deformity and scoliosis; as well as growth retardation, neurological disorder and hearing loss [2]. The average time spent on the ventilator was 3 days in our series. Any sequel depend to ventilator was not seen. However, severe GER developed in 2 patients. Antireflux surgery was done to these patients in the early period. In 3 patients, pectusexcavatum is available; however, operation will be decided during the adolescence period of these patients. We did not have any patient with neurological developmental delay on neurologic follow-up.

\section{CONCLUSION}

Bochdalek $\mathrm{CDH}$ management has shown significant improvement in the recent years. The survival rates after surgical repairs are increasing with the application of new treatment modalities with the advances in the NICU.

\section{Conflict of interest}

The authors disclosed no conflict of interest during the preparation or publication of this manuscript.

\section{Financing}

The authors disclosed that they did not receive any grant during conduction or writing of this study.

\section{REFERENCES}

1. Losty PD. Congenital diaphragmatic hernia: where and what is the evidence? Semin Pediatr Surg 2014;23:278-82.
2. Leeuwen L, Fitzgerald DA. Congenital diaphragmatic hernia. J Paediatr Child Health 2014;50:667-73.

3. Ameis D, Khoshgoo N, Keijzer R. Abnormal lung development in congenital diaphragmatic hernia. Semin Pediatr Surg 2017;26:123-8.

4. Puligandla PS, Grabowski J, Austin M, Hedrick H, Renaud E, Arnold M, et al. Management of congenital diaphragmatic hernia: A systematic review from the APSA outcomes and evidence based practice committee. J Pediatr Surg 2015;50:1958-70.

5. Benachi A, Cordier AG, Cannie M, Jani J. Advances in prenatal diagnosis of congenital diaphragmatic hernia. Semin Fetal Neonatal Med 2014;19:331-7.

6. Daodu O, Brindle ME. Predicting outcomes in congenital diaphragmatic hernia. Semin Pediatr Surg 2017;26:136-9.

7. McHoney M. Congenital diaphragmatic hernia, management in the newborn. Pediatr Surg Int 2015;31:1005-13.

8. Harting MT. Congenital diaphragmatic hernia-associated pulmonary hypertension. Semin Pediatr Surg 2017;26:147-53.

9. Beurskens LW, Schrijver LH, Tibboel D, Wildhagen MF, Knapen MF, Lindemans J, et al. Dietary vitamin A intake below the recommended daily intake during pregnancy and the risk of congenital diaphragmatic hernia in the offspring. Birth Defects Res A Clin Mol Teratol 2013;97:60-6.

10. Keller RL, Tacy TA, Hendricks-Munoz K, Xu J, Moon-Grady AJ, Neuhaus J, et al. Congenital diaphragmatic hernia: endothelin-1, pulmonary hypertension, and disease severity. Am J RespirCrit Care Med 2010;182:555-61.

11. Sbragia L, Nassr AC, Gonçalves FL, Schmidt AF, Zuliani CC, Garcia PV, et al. VEGF receptor expression decreases during lung development in congenital diaphragmatic hernia induced by nitrofen. Braz J Med Biol Res 2014;47:171-8.

12. Snoek KG, Reiss IK, Greenough A, Capolupo I, Urlesberger B, Wessel L, et al. Standardized Postnatal Management of Infants with Congenital Diaphragmatic Hernia in Europe: The CDH EURO Consortium Consensus - 2015 Update. Neonatology 2016;110:66-74.

13. Barroso C, Correia-Pinto J. Perioperative complications of congenital diaphragmatic hernia repair. Eur J Pediatr Surg 2018;28:141-7.

14. Maxwell D, Baird R, Puligandla P. Abdominal wall closure in neonates after congenital diaphragmatic hernia repair. J Pediatr Surg 2013;48:930-4.

15. Silen ML, Canvasser DA, Kurkchubasche AG, Andrus CH, Naunheim KS. Video-assisted thoracic surgical repair of a foramen of Bochdalek hernia. Ann Thorac Surg 1995;60:448-50. 